Pure Appl. Chem., Vol. 84, No. 12, pp. 2629-2639, 2012.

http://dx.doi.org/10.1351/PAC-CON-12-01-12

(C) 2012 IUPAC, Publication date (Web): 3 July 2012

\title{
Electronic interactions of silicon nanocrystals and nanocarbon materials: Hybrid solar cells*
}

\author{
Vladimir Švrček ${ }^{1, \ddagger}$ and Davide Mariotti ${ }^{2}$ \\ ${ }^{1}$ Research Center for Photovoltaic Technologies, AIST, Tsukuba, 305-8568, Japan; \\ ${ }^{2}$ Nanotechnology \& Integrated Bio-Engineering Centre (NIBEC), University of \\ Ulster, Newtownabbey, BT37 OQB, UK
}

\begin{abstract}
Hybrid inorganic/nanocarbon solar cells represent low-cost solutions for the largescale manufacturing of energy conversion devices. Here we discuss results that relate to the electronic interactions of nanocarbon materials with freestanding and surfactant-free silicon nanocrystals (Si-ncs) with quantum confinement effects, integrated in bulk-heterojunction solar cells. In particular, we demonstrate the feasibility of bulk-heterojunction photovoltaic solar cells that consist of Si-ncs combined with fullerenes or with semiconducting singlewalled carbon nanotubes (SWCNTs). We show that the energy levels between Si-ncs with energy gap exceeding $1.75 \mathrm{eV}$ and fullerenes are adequate for exciton dissociation and carriers (electrons/holes) generation and that hybrid solar cells formed by Si-ncs and semiconducting SWCNTs favor exciton dissociation only when a distinct chiral index [i.e., $(7,5)]$ is used. While fullerenes show energy conversion capabilities in the visible spectral region $(1.7-3.1 \mathrm{eV})$, the cells containing the SWCNTs, in comparison, have a considerably expanded optical response covering a broad range of the spectrum $(0.9-3.1 \mathrm{eV})$.
\end{abstract}

Keywords: electronic interactions; hybrid solar cells; silicon nanocrystals.

\section{INTRODUCTION}

Advances in the field of nanostructured photovoltaics have led to rapid improvements over the past decade in the power conversion efficiencies of this broad class of solar cells [1]. Building on the existing silicon technology and integrating new highly efficient nanostructured photovoltaics materials could represent a considerable advantage to achieve lower costs in the fabrication and installation of solar modules. For this reason, the manufacturing (e.g., low-temperature processing) and functional compatibility of many of the new nanomaterials is of paramount importance [2,3]. A particular advantage of nanostructured materials is the tunability of their optical and electronic properties, which may enable the implementation of "third-generation" approaches (i.e., strategies for reducing thermal losses) for improved photovoltaic power conversion efficiencies [4].

Hybrid solar cells, which combine advantages of both organic and inorganic semiconducting materials, could represent a considerable asset for the development of low-cost energy conversion technologies [5]. The potential of hybrid materials based on single-walled carbon nanotubes (SWCNTs), fullerenes $\left(\mathrm{C}_{60}\right)$, and silicon nanocrystals (Si-ncs) is not fully exploited yet [5,6]. Carbon-based nanomaterials, such as semiconducting SWCNTs and $\mathrm{C}_{60}$, have a range of useful properties with great

\footnotetext{
*Pure Appl. Chem. 84, 2499-2675 (2012). A collection of invited papers based on presentations at the $7^{\text {th }}$ International Conference on Novel Materials and their Synthesis (NMS-VII) and the $21^{\text {st }}$ International Symposium on Fine Chemistry and Functional Polymers (FCFP-XXI), Shanghai, China, 16-21 October 2011.

¥Corresponding author
} 
potential benefits for photovoltaics [7,8]. Namely, fullerenes are good candidates as an electron-accepting material in organic and hybrid solar cells, while semiconducting SWCNTs offer an enlargement of the absorption and conversion spectrum including the near-infrared region $(\sim 0.9 \mathrm{eV})$. Therefore, hybrid photovoltaic devices that consist of Si-ncs and nanocarbons might represent a unique combination of properties that can lead to efficient photon conversion.

Quantum confinement effects in Si-ncs have fueled a vibrant debate in the past two decades [9]. The use of $\mathrm{Si}$ as the main inorganic material is a great advantage due to its abundance in nature and a well-established Si-based photovoltaic industry. The demonstration of new physical phenomena in Si-ncs such as carrier multiplication opens up the possibility of achieving conversion efficiencies higher than $100 \%[10,11]$. For these reasons, the research activities addressing the role of quantum confinement effects in Si-ncs have dramatically increased, followed by considerable research efforts for the precision synthesis of Si-ncs and for their integration into photovoltaic devices [10,11]. The deployment of nanocarbon materials together with Si-ncs offers a combined set of novel physical phenomena for photon conversion, exploiting carrier multiplication within an enlarged spectral range [12].

The free-standing nature of Si-ncs facilitates the integration with nanocarbon materials in a bulkheterojunction architecture [12]. However, in order to achieve suitable optoelectronic device integration, it is important that Si-ncs remain in close proximity so that carrier transport can be facilitated. In this context, the use of self-assembled architectures that do not rely on surfactant-induced mechanisms seems to be advantageous. Contrary to surfactant- or molecular-induced self-assemblies, the spontaneous surfactant-free self-organization allows "constructing" percolating networks of Si-ncs without deteriorating the transport and charge collection that would be otherwise hindered by long molecules at the Si-ncs surface. In particular, Si-ncs that have been treated by a DC microplasma process or by ns-laser processing in liquid media have shown to form self-organized assemblies with the required characteristics $[13,14]$. In addition to the formation of spontaneous self-assemblies, surface engineering of Si-ncs contributes to enhancement of the electronic interactions with organic materials [15].

In this manuscript, we present results that relate to the electronic interactions and structural properties of newly developed composites based on free-standing Si-ncs with quantum confinement effects and nanocarbon materials. Furthermore, we report on the fabrication of hybrid solar cells that consist of Si-ncs and carbon-based nanomaterials, i.e., SWCNTs and fullerenes. We demonstrate the feasibility of bulk-heterojunction photovoltaic solar cells that consist of Si-ncs with fullerenes or with purified semiconducting SCNTs. The influence of the energy levels alignment on the formation of the bulkheterojunction is compared for both types of architectures, i.e., based on Si-ncs/fullerenes or based on Si-ncs/SWCNTs. We show that in all cases the combination of fullerenes and SWCNTs with free-standing Si-ncs leads to an efficient conversion together with a simplified approach for their integration in photovoltaic devices.

\section{EXPERIMENTAL DETAILS}

In order to obtain surfactant-free Si-ncs, boron-doped wafers with a resistivity of $\sim 0.5 \Omega \mathrm{cm}$ (p-type) were electrochemically etched along the crystallographic direction $\langle 100\rangle$ and subsequently mechanically pulverized, as described previously $[16,17]$. Subsequently, the larger grains were eliminated by sedimentation in ethanol for $30 \mathrm{~min}$. Our previous studies showed that Si-ncs are characterized by an average size of $\sim 3 \mathrm{~nm}$ and an average energy band-gap around $2 \mathrm{eV}$ [17].

In order to enhance the interactions within Si-ncs and allow formation of self-assemblies, 3D surface engineering by nanosecond (ns) laser processing in water was applied [18]. $2.5 \mathrm{mg}$ of the powder was dissolved in $10 \mathrm{ml}$ of water before being processed by the ns laser-fragmentation technique. The ns-pulsed laser process requires a homogeneous dispersion of the powder in the liquid media [18]. However, since the Si-ncs are hydrophobic, processing in water required the addition of a small amount of ethanol (10 drops) to wet the surface of the Si-nc aggregates and achieve a suitable homogeneous colloidal dispersion also in water. The fragmentation was conducted by irradiating the solution with a 
pulsed laser (Kr:F, $245 \mathrm{~nm}, 20 \mathrm{~Hz}, 130 \mathrm{~mJ} /$ pulse) at room temperature for $\sim 35 \mathrm{~min}$. The laser beam was focused onto a 3-mm-diameter spot on the liquid surface by a lens with a focal length of $250 \mathrm{~mm}$. During the irradiation, the glass container was closed and rotated.

Semiconducting SWCNTs were extracted from purchased carbon nanotubes powder (HiPCO, Carbon Nanotechnologies Inc.) using PFO (poly-9,9-di-n-octyl-fluorenyl-2,7-diyl, Sigma-Aldrich) as an extracting agent in toluene solution assisted by ultra-sonication and ultra-centrifugation techniques, as previously described [12]. In this study, the semiconducting SWCNTs sample consists of the following chiral indexes: $(7,5),(7,6),(8,6),(8,7)$, and $(9,7)[12]$. The diameter and optical band-gap of these semiconducting SWCNTs are in the range $0.8 \sim 1.1 \mathrm{~nm}$ and $0.8-1.1 \mathrm{eV}$, respectively.

To allow for a comparison, two types of hybrid solar cells were fabricated. The first type of solar cell was based on Si-ncs and fullerenes and was produced as follows. The Si-ncs were first subjected to $35 \mathrm{~min}$ of ns laser processing previously described. Following, 10 drops of poly(3,4-oxyethyleneoxythiophene):poly(styrenesulfonate) (PEDOT:PSS) were added to the processed Si-ncs colloid. The hybrid bulk-heterojunction solar cell device was fabricated by drop-casting the Si-ncs/PEDOT:PSS colloid on a glass substrate coated with indium tin oxide (ITO) and PEDOT $(\sim 150 \mathrm{~nm}$ ITO and $\sim 150 \mathrm{~nm}$ PEDOT thickness). After water evaporation, a 90-nm-thick fullerene film was deposited. Finally, 100 -nm aluminum electrodes were deposited through a shadow mask. The second type of solar cell was based on Si-ncs and purified semiconducting SWCNTs. For this second type of hybrid solar cell, a mixture of Si-ncs ( 2 mg) and SWCNTs $(\sim 0.01 \mathrm{mg})$ in $10 \mathrm{~mL}$ of toluene was sonicated and was then spraycoated on a PEDOT:PSS/ITO/glass substrate. The aluminum electrode (100 nm thick) was then deposited. The active area of both devices was $4 \mathrm{~mm}^{2}$. Finally, the devices were loaded in a measurement cell filled with nitrogen gas.

External quantum efficiency (EQE) of all the devices was measured under illumination with monochromatic light from a Xe lamp. Current-voltage $(I-V)$ characteristics of the cells were measured by using an AM1.5 light source simulated solar illumination at $100 \mathrm{~mW} \mathrm{~cm}{ }^{-2}$. The illumination intensity was calibrated by a standard a-Si solar cell.

\section{RESULTS AND DISCUSION}

\section{Free-standing surfactant-free Si nanocrystals with quantum confinement effects}

The typical room temperature photoluminescence (PL) spectrum as a function of energy for Si-ncs produced by electrochemical etching and dispersed in water is shown in Fig. 1. Under the quantum confinement model, reducing the crystal size causes a widening of the band-gap, which in this case reaches a value of about $2 \mathrm{eV}$. Quantum confinement in Si-ncs with sizes smaller than $5 \mathrm{~nm}$ also results in bright PL at room temperature. The inset of Fig. 1 shows a typical transmission electron microscope (TEM) image which confirms the Si-ncs to have diameters of about $3 \mathrm{~nm}$ and therefore with expected quantum confinement effects. However, the size distribution of the Si-ncs produced by electrochemical etching can be quite large and the resulting PL spectrum is therefore rather broad. The high PL intensity can be justified due to confinement effects in real space that would, most likely under Heisenberg's uncertainty principle, cause sufficient spreading of the wave-function in momentum space for direct band-to-band recombination, resulting in bright PL at room temperature [19]. At the same time, the effects of surface states influence the quantum confinement and PL and therefore surface characteristics have to be carefully considered to completely describe the behavior of Si-ncs. Although surface effects are not so easily explained, modified quantum confinement models are possible and required to account for all mechanisms [20,21]. 


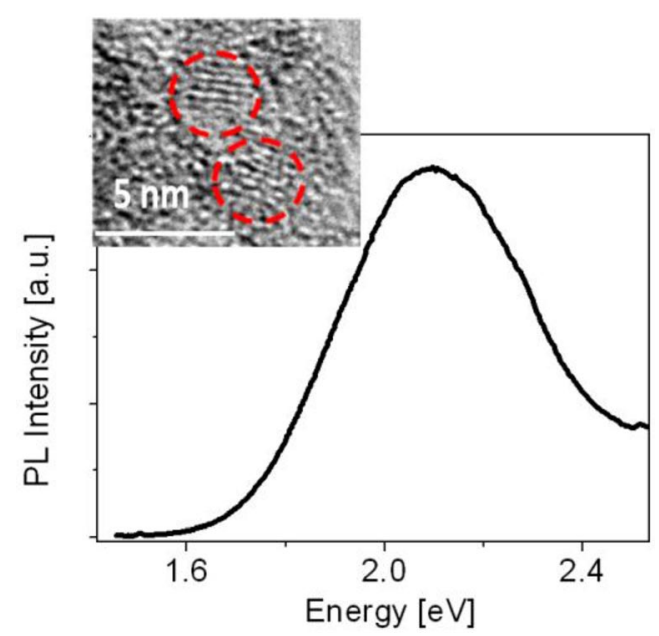

Fig. 1 Room temperature PL spectra as a function of energy for Si-ncs dispersed in water. Inset shows typical TEM image of Si-ncs produced by electrochemical etching.

One of the advantages of the electrochemical etching process is the high purity of the Si-ncs, which are virtually free from any contamination potentially originating from nonreacted products. Also, the resulting surface characteristics allow for further functionalization and properties tuning of Si-ncs at quantum confinement sizes. For instance, Si-ncs free of educts allow 3D surface engineering by DC microplasma or ns laser processing in liquid media, which results in the improvement of the PL intensity without using any surfactant [14]. Figure 2a presents a typical example of how the surface characteristics can be manipulated by 3D surface engineering induced by ns laser processing. The figure plots the PL intensity maxima as a function of the ns laser processing time $t$. It is observed that during the processing time up to $t=15 \mathrm{~min}$, the PL intensity increases (more then $40 \%$ ) and then as the process continues the intensity is reduced. The increase in PL intensity is due to the replacement of $\mathrm{H}$ surface bonds with $\mathrm{OH}$ bonds during the laser processing, which passivate the Si-ncs surface, removing any dangling bonds and surface defects [18]. Although $\mathrm{OH}$ terminations play a key role in the transformation of the Si-ncs surface characteristics, corresponding $\mathrm{OH}$ absorption is not observed in the Fourier transform infrared (FTIR) spectra because adjacent $\mathrm{OH}$ terminations tend to progress quickly to condensation and accelerated oxidation, producing water as a byproduct [18,22]. However, due to the $\mathrm{OH}$-induced oxidation, continuous ns laser processing introduces defects in the Si network, producing strained bonds [23] and resulting in the PL intensity decrease.

Generally, bulk Si presents hydrophobic surfaces, and also the majority of Si-ncs synthesis techniques produce Si-ncs with hydrophobic surfaces. As a result, their dispersion in aqueous solutions is rather difficult. Indeed, the ns laser interaction with the Si-ncs in water leads to peculiar physics, which results in the self-assembly of percolating conductive networks when the Si-ncs are dewetted on a substrate [14]. In this case, the self-assemblies are made from the solution, which shows quantum confinement effects and the PL properties at room temperature (Fig. 2, indicated by arrow). The self-assembly is the result of surface non-uniformity on the Si-ncs whereby the process in water contributes to surfactant-free Si-ncs and enhances the dipole-dipole interactions, which induces long-range selfassembly of connected networks.

In addition to enhancements of the dipole interactions between the Si-ncs, the OH-terminated Si-ncs also change the hydrophobic character of the surface into hydrophilic [15], which in principle allows the Si-ncs to be combined with water-soluble organic materials such as polymers. Water-soluble PEDOT:PSS dispersions are today one of the most promising and most widely used hole-injecting materials in organic-based photovoltaic devices and light-emitting diodes. Here we have therefore 

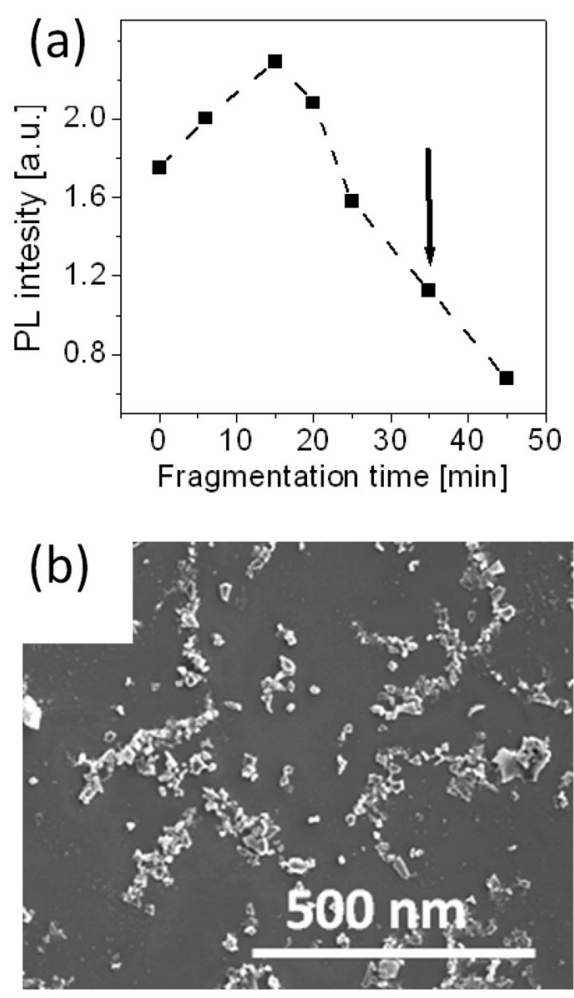

Fig. 2 (a) Summary of the PL intensity as a function of 3D surface engineering time induced by ns laser processing in water. (b) SEM image of dewetted Si-ncs: from the Si-nc/water/PEDOT:PSS colloid. A glass substrate with no patterning was used, and the solution was evaporated at ambient conditions.

investigated the water-based functionalization of 3D surface-engineered PL Si-ncs with PEDOT:PSS. As previously reported, we checked that the addition of PEDOT:PSS in the colloidal dispersion does not significantly affect the Si-ncs self-organization mechanism upon dewetting [15]. Figure $2 b$ shows typical SEM image of dewetted Si-ncs from the water/PEDOT:PSS-based dispersion. The self-assembled process could be repeated also in the presence of the polymer and patterns consisting of interconnected and conductive structures that exceeded several hundreds of micrometers in length $(>500 \mu \mathrm{m})$ were observed.

\section{Photovoltaic devices based on Si-ncs and nanocarbons}

Laser processing offers, therefore, the advantage of water solubility which allows a greater flexibility in producing Si-ncs/polymer (i.e., PEDOT:PSS) blends and consequently leads to enhanced opportunities to optimize the photovoltaic device performance [15,18]. Solution processing of Si-ncs with quantum confinement effects and engineered surface characteristics provide a low-cost and scalable technique for the fabrication of solar cells. Furthermore, solutions with engineered self-assembled and PEDOT:PSS-functionalized Si-ncs do not require a post-treatment to remove surfactants and/or ligands [17]. In order to demonstrate the photovoltaic action and the electronic interactions with nanocarbons, we fabricated bulk-heterojunction solar cells. While our current device structures and fabrication procedures are not yet optimized, they represent useful and simple arrangements that allow assessing the basic potential functionalities of the Si-ncs/nanocarbon system within a photovoltaic device. Figure 3 depicts the structures of the experimental solar cell devices. As shown in Fig. 3a, in the case of solar 


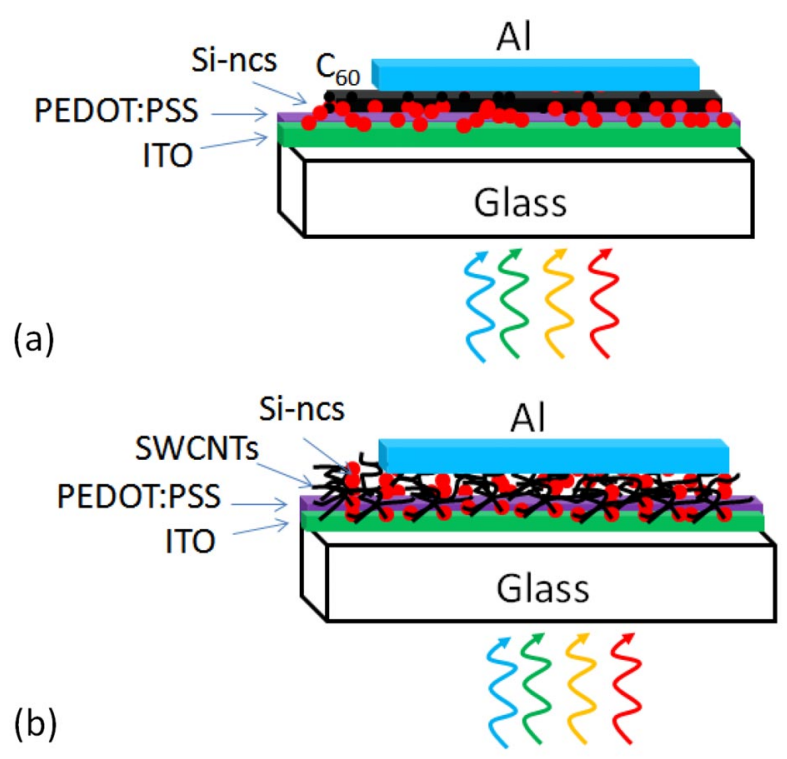

Fig. 3 Schematic diagram of the device structures representing the photovoltaic device based on (a) Si-ncs/C 60 and (b) Si-ncs/SWCNTs. Spin-coated PEDOT:PSS on ITO and alumina (Al) are used as electrodes.

cells based on $\mathrm{Si}-\mathrm{ncs} / \mathrm{C}_{60}$, the colloidal dispersion of Si-ncs has been drop-cast on the PEDOT layer of the ITO-coated glass substrate. The formation of the bulk-heterojunction with the Si-ncs has been achieved by depositing a fullerene on the dried Si-ncs/PEDOT:PSS assemblies. Figure $3 b$ shows the schematic structure of the hybrid solar cell that was made of a mixture of Si-ncs and SWCNTs and was spray-coated on PEDOT/ITO/glass substrate. Finally, in both cases, aluminum was deposited as counter electrodes.

The $I-V$ characteristics of the devices made out of the ns-laser engineered Si-nc and $\mathrm{C}_{60}$ are shown in Fig. 4a. The electrical characteristics confirm the presence of the heterojunction with a typical rectified diode characteristic with short-circuit current $\left(J_{\mathrm{SC}}\right)$ and open-circuit voltage $\left(V_{\mathrm{OC}}\right)$. Similarly, devices built with semiconducting SWCNTs and Si-ncs with quantum confinement effects show a rectification behavior (Fig. $4 \mathrm{~b}$ ), although, in this case, the rectification is poorer. The $I-V$ curve in Fig. 4b has an S-shape with the dark current flattening out near SC and at low forward bias. It has to be noted that hybrid solar cells consisting of only semiconducting SWCNTs exhibited a very small rectification behavior, a very small $J_{\mathrm{SC}}$ and $V_{\mathrm{OC}}$ [12]. The fill factor (FF) that describes the quality of the junctions is mainly influenced by the series and shunt resistances. The lower value observed for the devices with the SWCNTs (FF $\sim 25 \%$ ) is most likely due to the presence of the PFO polymer that was used during the separation of semiconducting from metallic nanotubes [12]. The low dark current suggests in both cases that the solar cells can operate and indicate that Si-ncs interact with SWCNTs and $\mathrm{C}_{60}$, respectively. Under AM1.5 irradiation, a photocurrent in both devices is generated. The $J_{\mathrm{SC}}$ is determined by the amount of the absorbed light. In particular, the prototypical hybrid solar cells based on Si-ncs and $\mathrm{C}_{60}$ or SWCNTs showed the following current characteristic: $J_{\mathrm{SC}}=0.24 \mathrm{~mA} / \mathrm{cm}^{2}$ and $J_{\mathrm{SC}}=0.3 \mathrm{~mA} / \mathrm{cm}^{2}$, respectively; it should be noted that neither the ratio between Si-ncs and PEDOT:PSS nor the thickness of the device is optimized in this stage of the investigation. 


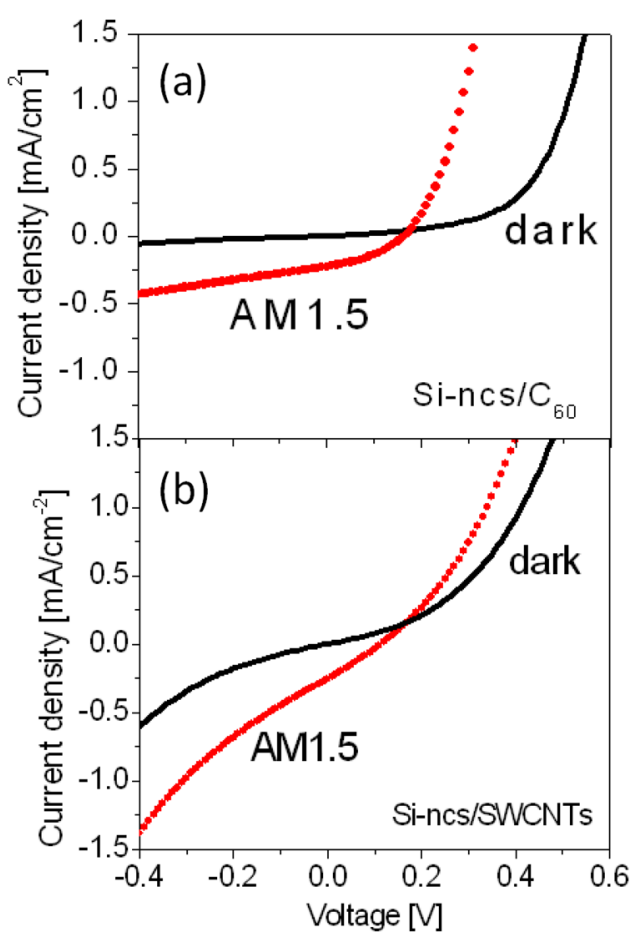

Fig. $4 I-V$ characteristics of solar cell devices produced with Si-ncs and $\mathrm{C}_{60}$ (a) or SWCNTs (b). $I-V$ characteristic in the dark (black line) and under AM1.5 illumination (gray line).

Figure 5 compares the normalized EQE as a function of the energy of the devices. Circles show the typical EQE of the device based on ns laser-engineered Si-ncs/PEDOT:PSS in water after the deposition of the $\mathrm{C}_{60}$ layer. The results indicate that closely packed Si-ncs/PEDOT:PSS assemblies can be electronically coupled with $\mathrm{C}_{60}$. As indicated by the $I-V$ characteristic of the device (Fig. $4 \mathrm{a}$ ), after the deposition of the $\mathrm{C}_{60}$ layer we have been able to form a bulk-like heterojunction solar cell. The interesting property is that the $\mathrm{EQE}$ in the visible region starts to increase from about $1.75 \mathrm{eV}$ where the absorption and electronic coupling of Si-ncs with $\mathrm{C}_{60}$ occurs (Fig. 5, indicated by arrow). In addition to the carrier generation and dissociation at the Si-ncs/fullerenes interface, the EQE is also determined and enhanced by the formation of percolation trajectories that allow for an improved carrier transport through the self-organized Si-ncs networks (Fig. 2b) [18]. The EQE in the visible range is mostly due to the absorption at the $\mathrm{C}_{60}$ layer.

In order to increase the generation of the photocurrent from the proposed type of hybrid nanocarbon-based solar cells, it is necessary to widen the absorption spectral range. One possibility is to use semiconducting SWCNTs which absorb light in the near-IR ranges $(<1.1 \mathrm{eV})$ with a consequent improved match of the solar spectrum. EQE of devices based on Si-ncs/SWCNTs is plotted by the black line in Fig. 5. Importantly, in the case of SWCNTs, the conversion active region is considerably enlarged (0.9-3.1 eV). Due to the absorption of the SWCNTs, the EQE shows an increased efficiency that goes beyond the infrared region. Enhanced EQE around 0.9-1.2 and 1.5-2 eV correspond to the optical transitions of semiconducting SWCNTs with the following chiral indexes: $(9.7),(8,6),(8,7)$, $(7,5),(7,6)$. We also observe a considerable increase in EQE ( $>2$ times) in the spectral region where the absorption of the Si-ncs overlaps $(\sim 1.2-2.7 \mathrm{eV})$ with that of SWCNTs if compared to the spectral range where the only SWCNTs contribution is expected (0.9-1.2 eV) [12]; these results clearly support that the electronic interactions between Si-ncs and SWCNTs were successfully achieved. 


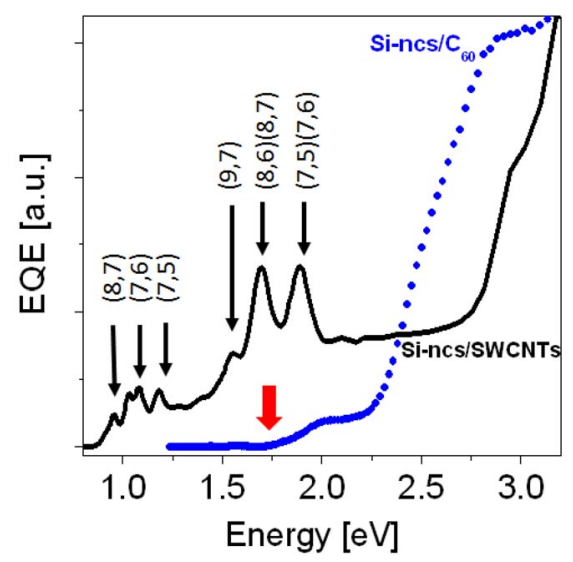

Fig. 5 Normalized EQE of a device based on Si-ncs/C 60 (dots) and of the device based on Si-ncs/SWCNTs (black line). Si-ncs were surface engineered by ns laser in water and functionalized with PEDOT:PSS.

Figure 6 reports the corresponding band diagrams of our devices. Figure $6 a$ depicts the energy levels for a device based on Si-ncs, fullerenes, Al, PEDOT:PSS, and Fig. $6 \mathrm{~b}$ for a similar device based on semiconducting SWCNTs with different chiral indexes $(\mathrm{n}, \mathrm{m})$. Hybrid solar cells that consist of Si-ncs and both types of nanocarbons have given evidence of the formation of a bulk heterojunction. Because the size distribution of the Si-ncs is relatively broad, Fig. 6a depicts multiple energy levels above about $-4.1 \mathrm{eV}$ (bulk conduction band-edge) up to about $-3.55 \mathrm{eV}$, which would result from the quantum confinement induced band-gap widening. The conduction band of Si-ncs can be up to $-3.55 \mathrm{eV}[12,15]$, which is higher than any of the $\mathrm{C}_{60}$ lowest unoccupied molecular orbital (LUMO) values reported in the literature [18] and allows the formation of a bulk heterojunction. Excitons are created in this case by photon absorption in both the Si-ncs and fullerenes. Then, the difference in electron affinity and ionization potential between the nanocrystals and fullerenes provide the energy driver for the dissociation of excitons and consequent photoconductivity generation. Similarly to the fullerenesbased device, exciton dissociation in the Si-ncs/SWCNTs devices can be explained by comparing the respective energy levels, Fig. 6b. Depending on the chiral index of the SWCNTs, the electron affinity and ionization potential are in the range 3.98-4.1 and 4.85-5 eV, respectively [12]. The excess of holes in p-type Si-ncs (we used p-type doped $\mathrm{Si}$ wafer for Si-ncs fabrication) shifts up the respective conduction and valence bands after Fermi levels equalization [12]. Consequently, the energy levels of the Si-ncs and SWCNTs improve their respective alignment, allowing for the formation of a bulk-type heterojunction. The offsets are sufficient for exciton dissociation and are consistent with our observations of electron transfer from the Si-ncs to the $\mathrm{C}_{60}$ or the SWCNTs and hole transfer to the Si-ncs. In both solar cells, the Si-ncs serve as hole-transporting material when electronically coupled with an electron-accepting semiconducting SWCNTs or fullerenes.

The band-gap limits the splitting of the quasi Fermi level of electrons and holes, induced by the light absorption. In our devices, instead of the band-gap, the energy separation between the highest occupied molecular orbital (HOMO) of the donor and the LUMO of the acceptor has to be considered as the limitation for the $V_{\mathrm{OC}}$. The thermodynamic limit for the $V_{\mathrm{OC}}$ is influenced by the band-gap of the active materials, which is almost the same for both devices. Namely, $V_{\mathrm{OC}}$ is about $0.16 \mathrm{~V}$ for $\mathrm{Si}-\mathrm{ncs} / \mathrm{C}_{60}$ and about $0.14 \mathrm{~V}$ for devices based on Si-ncs/SWCNTs. For the positive contact, where ITO-coated glass with PEDOT:PSS is regularly used, a strong dependence of the $V_{\mathrm{OC}}$ on the work function of the PEDOT:PSS electrode was demonstrated in addition to a dependence on the HOMO level. The bandgap of Si-ncs with quantum confinement effects $(>1.7 \mathrm{eV})$ will give a conduction band-edge higher than $3.7 \mathrm{eV}$, which is the LUMO of fullerenes and might allow the formation of type-II bulk heterojunction. 

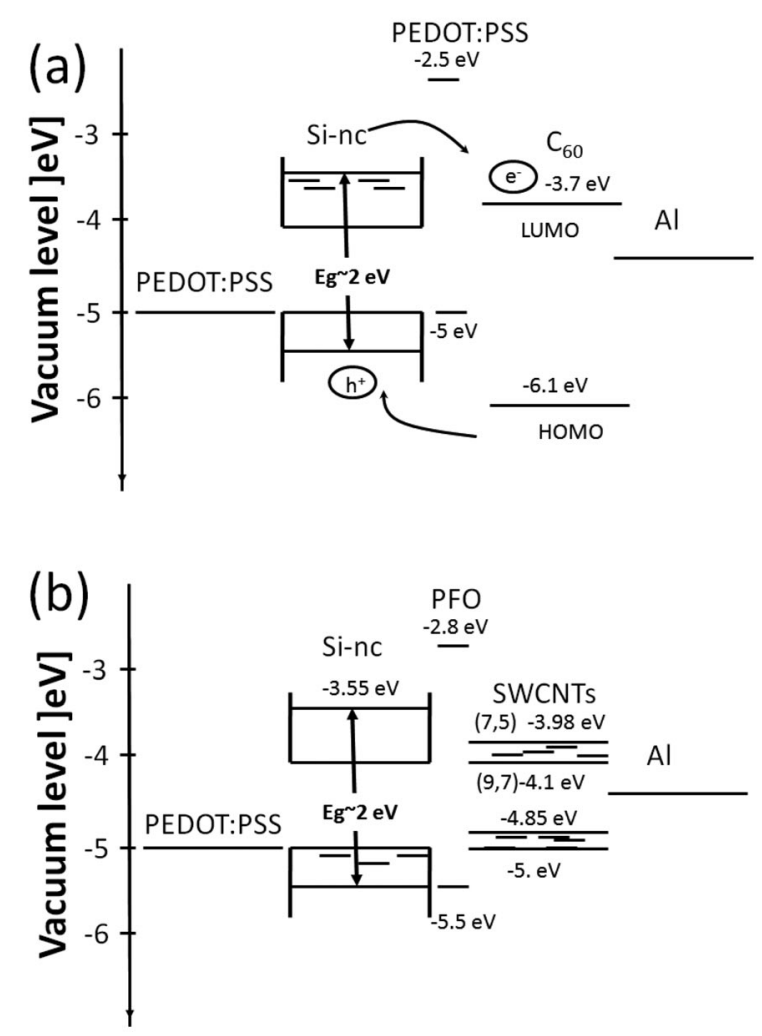

Fig. 6 Energy levels of (a) $\mathrm{C}_{60}$ Si-ncs with quantum confinement, Al, ITO, and PEDOT:PSS and of (b) semiconducting SWCNTs with different chiral indexes are shown.

This corroborates our observations since a clear higher EQE is recorded in the range above $1.7 \mathrm{eV}$ (Fig. 5, blue circles). Furthermore, PEDOT:PSS is an excellent hole-injection material that facilitates hole transfer and at the same time serves as good electron-blocking layer (Fig. 6a). Exciton dissociation has then contributed to produce negatively charged Si-ncs where electrons are effectively blocked at the PEDOT:PSS shell until successful electron transport to the fullerene film is achieved with low recombination chances.

In the case of the devices based on Si-ncs/SWCNTs, the electronic structure and the energy levels depend additionally on the chiral index $(n, m)$ of the semiconducting SWCNTs. The electron affinity and ionization potential of $(7,5),(7,6),(8,6),(8,7)$, and $(9,7)$ SWCNTs are in the range from 3.98 to $4.1 \mathrm{eV}$ and from 5 to $4.85 \mathrm{eV}$ (Fig. 6). Due to the equalization of the Fermi levels between p-type Si-ncs and SWCNTs, the energy levels are shifted, which enhances holes transfer into Si-ncs [12]. In this case, the $\mathrm{Si}$-nc conduction band is about $-3.55 \mathrm{eV}$ and the band-gap $\sim 2.0 \mathrm{eV}$ with the valence band around $-5.55 \mathrm{eV}$. A type-II heterojunction in this case is probably unlikely and a type-I heterojunction is more probable. However, it cannot be excluded that the nanotubes and nanocrystals are not interacting at all with each other and that each material is only interacting with the PEDOT:PSS and the Al electrodes, independently, giving some weak exciton dissociation. The optical band-gap $=2.0 \mathrm{eV}$ is approximately evaluated at the maximum of the PL spectra. An optical band-gap as low as $1.15 \mathrm{eV}$ will give a valence band-edge as low as $4.55 \mathrm{eV}$, which might allow for type-II heterojunction, e.g., for SWCNTs that have chiral index $(7,5)$. The optical absorption peak intensities of $(7,5)$ and $(7,6)$ are smaller than those of $(9,7),(8,7)$, and $(8,6)$, but the EQE peak intensity is just the opposite. Therefore, the EQE and exciton dissociation yield of $(7,5)$ and $(7,6)$ is larger than that of $(8,7),(8,6)$, and $(9,7)$ SWCNTs [12]. In addi- 
tion to the band structure, which is consistent with our interpretation, we have further evidence of the formation of a type-II heterojunction. Firstly, solar cells consisting only of either Si-ncs or SWCNTs show very poor diode characteristics, whereas their combination shows a synergic effect. Secondly, the PL intensity of the Si-ncs is quenched by the addition of semiconducting SWCNTs [12]. Thirdly, it is important to highlight that the enhancement of the charge generation with the composite p-type Si-ncs/SWCNTs is by orders of magnitude, if compared to n-type Si-ncs/SWCNTs [12]. Our results also suggest that the photocarrier generation intrinsically involves both SWCNTs and Si-ncs, and it is not due to the formation of a Schottky-type junction with the electrodes. Admittedly, further investigations will be still needed to fully confirm our analysis and unambiguously conclude on the type of the heterojunction.

Finally, both types of devices will require separate optimization of the thickness. The variation of the EQE with the thickness is expected to be a general feature of thin-film photovoltaic devices, which is limited by transport/recombination processes. The spatially varying optical field in both types of devices is also very different. For example, the majority of the absorption in the devices with the nanotubes will occur near the PEDOT:PSS, since the light will not significantly penetrate into the films of nanotubes. In contrast, the blended devices will observe increased penetration, in particular at wavelengths where the nanocrystal absorption is spectrally weaker. An ideal situation would result whereby the absorption coefficient can be made to grow linearly with the wavelength. This also suggests that tandem devices with two absorbers optimized for short- and long-wavelength regions may offer an interesting alternative to make more efficient hybrid solar cells absorbing in a large spectral region.

\section{CONCLUSION}

We have demonstrated the feasibility of type-II bulk-heterojunction photovoltaic solar cells made from surfactant-free Si-ncs with quantum confinement effects combined with fullerenes $\left(\mathrm{C}_{60}\right)$ and with purified semiconducting SWCNTs. In the prototype solar cells, an electronic interaction between Si-ncs and both nanocarbon materials has been confirmed. In both cases, we have shown that Si-ncs with quantum confinement sizes $(\sim 3 \mathrm{~nm})$ can serve as electron-transporting material and where $\mathrm{C}_{60} / \mathrm{SWCNTs}$ behaves as hole-transporting material. We argue that in the case of nanotubes the chirality plays an important role for the formation of a given type of the bulk heterojunction. The bulk-heterojunction photovoltaic solar cells consisting of SWCNTs and Si-ncs suggest that the combination of Si-ncs and semiconducting SWCNTs such as $(7,5)$ is electronically favorable for exciton dissociation and carrier (electrons/holes) generation. Importantly, in the case of SWCNTs, a conversion efficiency region is considerably enlarged (0.9-3.1 eV). We believe that the combination of Si-ncs with nanocarbon materials such as $\mathrm{C}_{60}$ or semiconducting SWCNTs is a viable and promising approach for low-cost, environmentally friendly, and efficient solar cells. Furthermore, investigations of Si-ncs/nanocarbons interactions are today largely unexplored, and room for further improvements certainly exists.

\section{ACKNOWLEDGMENTS}

This work was partially supported by a NEDO Project (Japan) and by D.M.'s JSPS Invitation Fellowship (Japan).

\section{REFERENCES}

1. M. A. Green, K. Emery, Y. Hishikawa, W. Warta. Prog. Photovoltaics 19, 84 (2011).

2. S. R. Forrest. MRS Bull. 30, 28 (2005).

3. C. J. Brabec, N. S. Sariciftci, J. C. Hummelen. Adv. Funct. Mater. 11, 15 (2001).

4. M. A. Green. Prog. Photovoltaics 9, 123 (2001).

5. D. J. Milliron, I. Gur, A. P. Alivisatos. MRS Bull. 30, 41 (2005).

(C) 2012, IUPAC

Pure Appl. Chem., Vol. 84, No. 12, pp. 2629-2639, 2012 
6. S. Iijima. Nature 354, 56 (1991).

7. N. S. Sariciftci, L. Smilpwitz, A. J. Heeger, F. Wudl. Science 258, 1474 (1992).

8. M. Gabor, Z. Zhong, K. Bosnick, J. Park, P. L. McEuen. Science 325, 1367 (2009).

9. L. T. Canham. Appl. Phys. Lett. 57, 1046 (1990).

10. M. C. Beard, K. P. Knutsen, P. R. Yu, J. M. Luther, Q. Song, W. K. Metzger, R. J. Ellingson, A. J. Nozik. Nano Lett. 7, 2506 (2007).

11. A. J. Nozik. Chem. Phys. Lett. 457, 3 (2008).

12. V. Švrček, S. Cook, S. Kazaoui, M. Kondo. J. Phys. Chem. Lett. 2, 1646 (2011).

13. V. Švrček, D. Mariotti, M. Kondo. Appl. Phys. Lett. 97, 161502 (2010).

14. V. Švrček, D. Mariotti, K. Kalia, M. Kondo. Chem. Phys. Lett. 478, 224 (2009).

15. V. Švrček, D. Mariotti, T. Nagai, Y. Shibata, I. Turkevych, M. Kondo. J. Phys. Chem., C 115, 5084 (2011).

16. V. Švrček, A. Slaoui, J.-C. Muller. J. Appl. Phys. 95, 3158 (2004).

17. V. Švrček, T. Yamanari, Y. Shibata, M. Kondo. Acta Mater. 59, 764 (2011).

18. V. Švrček, D. Mariotti, Y. Shibata, M. Kondo. J. Phys. D: Appl. Phys. 43, 415402 (2010).

19. A. G. Cullis, L. T. Canham. Nature 353, 335 (1991).

20. A. G. Cullis, L. T. Canham, P. D. J. Calcott. J. Appl. Phys. 82, 909 (1997).

21. D. J. Lockwood, G. C. Aers, L. B. Allard, B. Bryskieicz, S. Charbonneau, D. C. Houghton, J. P. McCaffrey, A. Wang. Can. J. Phys. 70, 1184 (1992).

22. Y. H. Ogata, F. Kato, T. Tsuboi, T. Sakka. J. Electrochem. Soc. 145, 2439 (1998).

23. J. S. Chou, S. C. Lee. J. Appl. Phys. 77, 1805 (1995). 